\title{
Acidente Vascular Cerebral por dissecção carotídea bilateral pós-procedimento odontológico: relato de caso
}

Oscar Reimann Junior ${ }^{1}$ (D), Heraldo de Oliveira Mello Neto ${ }^{2}$ (D), Louise Caroline Azevedo Ferreira ${ }^{3}$ (D), Jonathan Wei Ting Wen Liu ${ }^{4}$ (D), Chelin Auswaldt Steclan ${ }^{5}$ (D), Adriélle da Costa $^{5}$ (D), Michael Ricardo Lang ${ }^{6}$ (D)

\begin{abstract}
RESUMO
Introdução: A dissecção da artéria carótida (DAC) tem como uma das principais repercussões o Acidente Vascular Cerebral isquêmico ( $\mathrm{AVCi}$ ) em indivíduos jovens previamente saudáveis. É comum que nas DAC traumáticas, como em acidentes automobilísticos, o paciente seja submetido à investigação mais complexa com exames de imagem. Estes permitem um diagnóstico precoce e, portanto, um tratamento, reduzindo as chances de sequelas. Todavia, causas não traumáticas com desfecho de DAC já foram relatadas, como as causas odontológicas. Objetivo: Contribuir para valorização diagnóstica da DAC não traumática e profilaxia de AVC subsequente. Métodos: Relatamos um caso de dissecção não aneurismática das artérias carótidas internas (ACI) pós-procedimento odontológico com desfecho de AVC bilateral em paciente de 52 anos. Resultados: Devido à forte associação de DAC a fatores traumáticos, em um primeiro contato com a paciente não foram levantadas suspeitas da ocorrência de DAC nem de AVC (ausência de déficit focal). Porém, tardiamente, foi identificada oclusão da ACI à esquerda e estenose na ACI direita, resultando em dois AVCs e incapacidade funcional severa na alta. Conclusão: O diagnóstico da DAC é um desafio, em especial por tratar-se de uma lesão de etiologia multifatorial. No entanto, seu reconhecimento precoce afetará diretamente o desfecho do paciente. Portanto, na presença de eventuais sintomas de alerta, faz-se necessário um meticuloso questionamento sobre as últimas atividades do indivíduo. Este estudo alerta para a adoção de condutas profiláticas na ocorrência de fatores causais como hiperextensão cervical prolongada ou movimentos súbitos da cervical.
\end{abstract}

Palavras-Chave: Dissecação da artéria carótida interna, Acidente vascular cerebral, Implantação dentária.

\section{INTRODUÇÃO}

A dissecção da artéria carótida (DAC) tem como uma das principais repercussões o Acidente Vascular Cerebral isquêmico (AVCi) ${ }^{1}$. Com uma incidência em torno de $2 \%$ dos AVCs na população em geral, essa porcentagem aumenta para 10 a $20 \%$ quando considerada a faixa etária abaixo de 50 anos $^{2}$. A fisiopatologia dos mecanismos isquêmicos secundários à DAC relaciona-se à estenose luminal com isquemia distal por hipoperfusão encefálica quando aneurismática ou mecanismos embólicos ${ }^{3}$. No entanto, estudos relacionados aos potenciais fatores de risco, a apresentação dos sintomas e ao tratamento da DAC são dependentes do tipo de dissecção, com pouco consenso entre eles ${ }^{4,5}$.

As DACs de aparecimento espontâneo não são associadas a traumas graves (ex. automobilísticos) e sim, a alterações estruturais da artéria (distúrbios hereditários da parede e do tecido conjuntivo $)^{5}$ ou a traumas menores na cervical ${ }^{4}$, tais como hiperextensão do pescoço ${ }^{6}$, manipulações cervicais ${ }^{7}$, ou ainda, relacionam-se com doenças infecciosas ${ }^{8}$. Há controvérsias do envolvimento de fatores de risco vasculares como hipertensão e hipercolesterolemia nesse tipo de ocorrência9 .

Procedimentos odontológicos podem estar associados a fatores causais de DAC pela simples

Hospital São Vicente de Paulo, Mafra, (SC), Brasil.

Clínica X-LEME Diagnóstico por Imagem, Curitiba, (PR), Brasil.

Hospital São Vicente de Paulo, Mafra, (SC), Brasil.

Hospital Universitário Cajuru. Serviço de Neurologia, Curitiba, (PR), Brasil.

Universidade do Contestado (UnC). Escola de Medicina, Mafra, (SC) Neuromax, Brasil.

Universidade do Contestado (UnC). Hospital São Vicente de Paulo. Escola de Medicina, Mafra, (SC) Neuromax, Brasil. 
manutenção da postura em hiperextensão cervical prolongada com paciente anestesiado durante o procedimento dentário ${ }^{10}$. Ao nosso conhecimento, até o momento, somente quatro estudos relataram a ocorrência da DAC após procedimento odontológico, com ocorrência bilateral ${ }^{10-12}$ e unilateral, resultando em $\mathrm{AVC}^{8}$.

Com tantos fatores causais frequentemente não considerados e um quadro sintomático insidioso, pois a lesão carotídea pode apresentar diferentes sinais clínicos no momento da dissecção das artérias cervicais ${ }^{9}$, o resultado pode ser um diagnóstico tardio refletindo no prognóstico. A repercussão da DAC em AVCi só pode ser confirmada pela visualização direta da lesão encefálica e estreitamento ou oclusão do lúmen da artéria cervical através de exames de imagem. A angiografia demonstra o lúmen arterial e mostra irregularidades da parede ${ }^{13}$. Embora esta seja a modalidade padrão ouro para o diagnóstico de DAC, a angiorressonância magnética (angioRM) e/ou angiotomografia computadorizada (angioTC) são alternativas não invasivas e atualmente são métodos de neuroimagem preferenciais ${ }^{13}$.

Considerando tais informações, e visando contribuir para valorização do diagnóstico, descrevemos um caso de disseç̧ão não aneurismática das carótidas internas à esquerda e a direita, resultando em AVC bilateral e incapacidade funcional severa na alta. Os mecanismos etiopatogênicos desse caso singular de DAC são amplamente discutidos e comparados com os principais registros da literatura.

\section{RELATO DE CASO}

Paciente do sexo feminino, 52 anos, hipertensa e tabagista, procurou o serviço de emergência com quadro de desconforto na região cervical esquerda e dificuldade para elevar o braço esquerdo. Os sintomas iniciaram após procedimento odontológico de extração e implante dentário com horas de duração. Ao exame inicial, apresentava-se consciente, orientada, deambulando normalmente, pressão arterial: 140/80 $\mathrm{mmHg}$, afebril, dor supraescapular. Força motora grau 3 para abdução do braço e flexão do ombro esquerdo, grau 5 nos demais grupos musculares e membros. Encarado inicialmente como dor os- teomuscular secundária ao procedimento odontológico (extração de quatro dentes e implante), o qual foi inicialmente tratado com analgésicos comuns e recebendo alta do pronto atendimento.

A paciente retornou no dia seguinte com piora do quadro, apresentando hemiparesia esquerda desproporcionada com predomínio facio-braquial proximal, pressão arterial: 160/80 $\mathrm{mmHg}$, alerta, Escala de Coma de Glasgow: 15, sem desvio do olhar conjugado, pontuou 5 na National Institute of Health Stroke Scale (NIHSS). A Tomografia Computadorizada (TC) de crânio de emergência demonstrou hipodensidade cortical em região parieto-occipital do hemisfério direito sugestiva de lesão isquêmica aguda. Na ocasião, não foi realizado trombólise com Ativador do Plasminogênio Tecidual (rTPA) ou trombectomia mecânica, pois já se encontrava fora da janela terapêutica. Prontamente foram iniciadas as medidas de suporte para o AVCi e solicitado exames para investigação etiológica.

Enquanto aguardava os exames complementares, a paciente evoluiu com crises convulsivas reentrantes (estado de mal convulsivo) refratária às medidas iniciais, necessitando de intubação orotraqueal e suporte ventilatório. A avaliação comparativa com resultados de uma segunda TC de crânio demonstrou novas hipodensidades, comprometendo todo território irrigado pela artéria cerebral média (ACM) esquerda (Figura1).

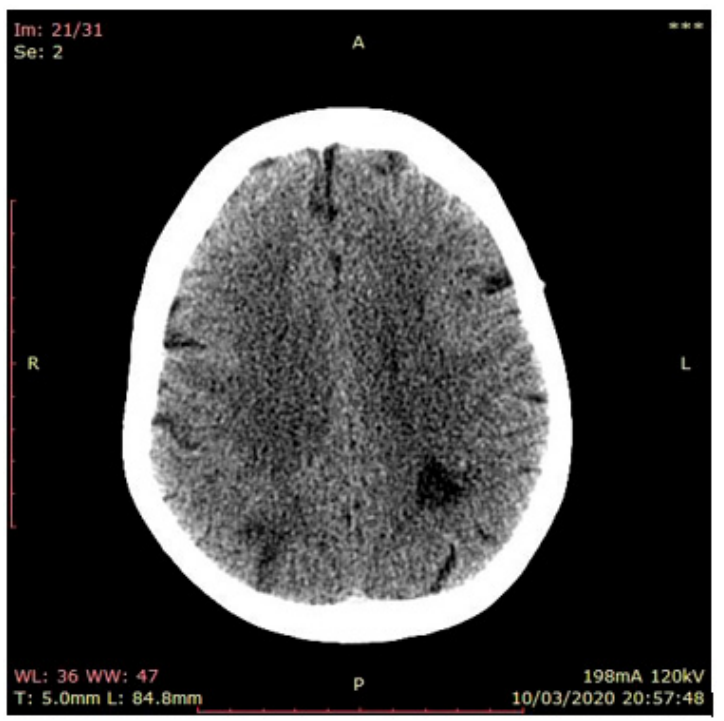

Figura 1: TC de Crânio no plano axial evidenciando hipodensidades em região parieto-occipital do hemisfério direito (insulto isquêmico tipo watershed) e em território da Artéria Cerebral Média (ACM) esquerda. 
Em virtude do quadro, foi realizado ecocardiograma transtorácico na beira do leito que não mostrou fontes emboligênicas. O Doppler de carótidas e vertebrais mostrou oclusão da artéria carótida comum esquerda sem alterações na circulação carotídea direita e nas vertebrais. A angioTC de crânio e cervical mostrou oclusão de artéria carótida interna (ACI) à esquerda e estenose na ACI direita, ambas com características compatíveis com DAC (Figura 2) e redução acentuada de vascularização no hemisfério esquerdo, sendo estes supridos pelas anastomoses (Círculo de Willis) e colaterais (Figura 3).

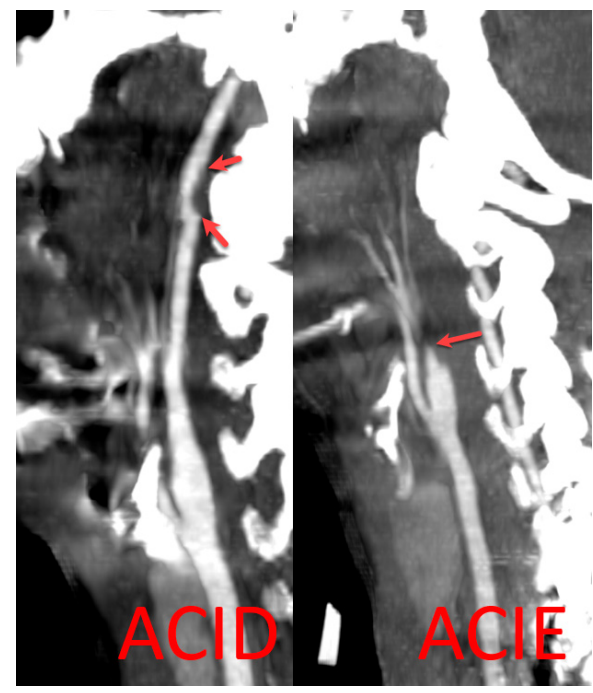

Figura 2: Corte sagital de Angiotomografia Cervical demonstrando estenose e oclusão das Artérias Carótidas Internas no seu segmento extracraniano (ACID: Artéria Carótida Interna Direita; ACIE: Artéria Carótida Interna Esquerda).

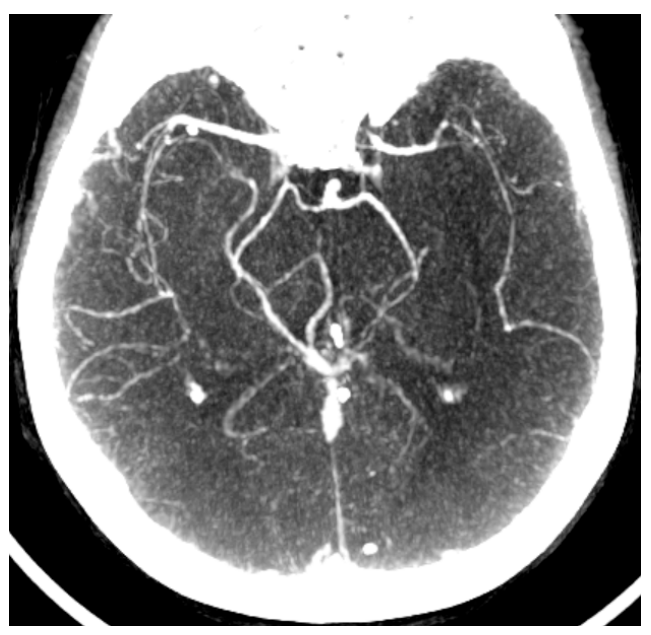

Figura 3: Angiotomografia de crânio em corte axial demostrando redução acentuada de vascularização no hemisfério esquerdo e preenchimento dos vasos pelo contraste através de anastomoses e colaterais.
A pesquisa diagnóstica restante com eletrocardiograma evidenciou ritmo sinusal e sem sinais de isquemia. Os exames laboratoriais sem alterações notáveis. A paciente foi encaminhada para Unidade de Terapia Intensiva (UTI) onde ficou por 35 dias, persistindo com estado de mal refratário.

Recebeu alta da UTI em estado minimamente consciente e totalmente dependente apresentando escore 5 na Escala de Rankin (ERm). Após cinco meses da alta, no seguimento ambulatorial (follow-up) a paciente não apresentou novos AVCs. Porém, encontra-se ainda com incapacidade severa (ERm 5 em 3 de agosto do ano corrente). Este estudo foi aprovado pelo Comitê de Ética em Pesquisa da instituição proponente (n. ${ }^{0}$ 3.609.943) e o responsável legal assinou o Termo de Consentimento Livre e Esclarecido autorizando o relato de caso.

\section{DISCUSSÃO}

Nosso estudo apresentou o relato de caso de uma paciente com episódio de DAC bilateral após procedimento odontológico, com apresentação inicial de cervicalgia e déficit motor em ombro, ambos à esquerda. Tardiamente, houve evolução neurológica grave devido a dois AVCs com consequente hemiparesia e incapacidade funcional severa na alta. Foi identificada oclusão na CI esquerda com lesão cerebral em região entre ACM e artéria posterior direita. Sabe-se que, quando há o envolvimento das camadas mais internas dos vasos (íntima e média) ocorre estenose luminal, promovendo isquemia distal por hipoperfusão' ${ }^{9}$. A paciente ainda apresentou uma estenose na artéria CI direita com isquemia no território da ACM esquerda compatível com embolia.

Na primeira visita da paciente ao serviço de emergência, os únicos sinais eram a cervicalgia e déficit na elevação do ombro. Assim, a ausência de outros sinais sugestivos de DAC conduziu a um diagnóstico inicial de dor osteomuscular pelo próprio posicionamento intraoperatório. De acordo com a literatura, outros sinais sugestivos são aqueles secundários aos eventos isquêmicos, que incluem a associação de perda de consciência, hemiparesia, afasia e síndrome de Horner, com 
história prévia na maioria das vezes de traumatismo cervical ${ }^{9,14}$. No entanto, estudos demonstram que a associação destes sinais está presente em apenas um terço dos casos de $\mathrm{DAC}^{5,9}$, podendo ocorrer isoladamente, ou mesmo a dissecção ser assintomática ${ }^{3,5,9}$. É comum que nas DAC traumáticas, como em acidentes automobilísticos, o paciente seja submetido à investigação mais complexa com exames de imagem que acabam por diagnosticar precocemente as DACs, as quais são tratadas antes que o déficit neurológico se instale $^{1}$. No entanto, o mesmo não ocorre na DAC espontânea ou por traumas menores, como na paciente em questão, predispondo a imprecisão diagnóstica inicial, pois, além do fator causal não levantar a hipótese diagnóstica, a paciente no primeiro momento não apresentava nenhum déficit focal, diferentemente do estudo retrospectivo de Campos et al. ${ }^{15}$, em que todos os pacientes apresentaram déficit focal.

Entretanto, mesmo com a presença de sinais clínicos associados sugestivos da DAC, o diagnóstico só é comprovado pela visualização direta do hematoma intramural e estreitamento ou oclusão arterial do lúmen ${ }^{13}$. A questão mais frequente diz respeito à realização de exames invasivos diante da suspeita DAC, como a angiografia para a indicação cirúrgica ou se métodos não invasivos como o ultrassom (US) modo $B$, seriam suficientes ${ }^{13}$. Com a AngioRM e a AngioTC, a questão sobre o melhor exame de imagem para diagnosticar a DAC ficou ainda mais complexa. A maioria dos centros terciários iniciam a investigação com AngioRM e/ou AngioTC, reservando angiografia (Padrão Ouro) para casos inconclusivos.

O problema da realização de exames de imagem é o alto custo, o que restringe a realização pelo Sistema Único de Saúde (SUS). É cabível que se não há fatores iniciais que motivem uma investigação nesse âmbito, estes acabam não sendo realizados, fato este, que afeta diretamente a investigação, e consequentemente, a confirmação do diagnóstico. Porém, sabendo que o custo de um paciente pós AVC para o SUS é em média $\mathrm{R} \$ 6 \mathrm{mil}$, com custo de $\mathrm{R} \$ 640$ por dia em casos de sequelas leves a $R \$ 32$ mil para pacientes com sequelas graves e períodos de internação de um mês, o diagnóstico precoce da DAC pode minimizar os eventos neurológicos e até prevenir
- AVC, diminuindo também a sobrecarga econômica ao serviço de saúde do país ${ }^{16}$.

A paciente apresentou hemiparesia pronunciada vinte e quatro horas mais tarde, que foi verificada no retorno ao serviço de emergência. Segundo a literatura, os intervalos entre a DAC e o aparecimento dos sinais e sintomas sugestivos da injúria podem ser variáveis ${ }^{3,15}$, pois a dissecção pode estar em curso. Diante disso, a evolução da gravidade no quadro clínico, provavelmente, deu-se pelo aumento do flap com diminuição progressiva do lúmen arterial ${ }^{9}$. O fluxo foi reduzindo causando um infarto cerebral progressivo iniciando pela periferia (infarto tipo "watershed"). Tendo isso, pela falta de sinais clínicos para o estabelecimento de um diagnóstico precoce, a "janela terapêutica" foi perdida, o que provavelmente ocasionou o aumento da zona central isquêmica com o recrutamento de células da zona de penumbra9.

Considerando o fator causal da DAC, vários detalhes anamnésicos de hiperextensão ou rotação repentina do pescoço frequentemente precedem o desenvolvimento da DAC. Movimentos repentinos da cervical podem ferir mecanicamente a parede arterial ${ }^{17}$, inclinação prolongada da cabeça ${ }^{18}$, atividades esportivas ${ }^{19}$, manipulações cervicais ${ }^{7}$, procedimentos médicos e cirúrgicos como anestesia ${ }^{20}$. No caso da paciente em questão, o procedimento odontológico pode ser etiologicamente considerado ${ }^{10,11}$ devido à prolongada inclinação do pescoço e aos movimentos súbitos da cervical pela extração dentária. A abertura da boca reduz a distância entre o ângulo da mandíbula e as vértebras cervicais superiores favorecendo a compressão das artérias carótidas cervicais contra os processos transversos das vértebras cervicais superiores ou outras regiões ósseas ${ }^{17}$. O alongamento mecânico dos tecidos moles do pescoço também pode causar a ruptura da túnica íntima e média com preservação da adventícia mais elástica ${ }^{21}$. Assim, essas são hipóteses dos mecanismos da DAC a serem consideradas.

Casos semelhantes após procedimento odontológico já foram relatados anteriormente ${ }^{8,10-12}$. Dois deles apresentaram associação de infecção periodontal com a DAC, porém, em nosso estudo, dados os resultados dos exames laboratoriais normais, a possibilidade do envolvimento de processos infecciosos pode ser descartada. No caso retrata- 
do por Cerrato et al. (2004), a paciente apresentou comprometimento sensório-motor no membro superior (MS) e hemianopsia inicial devido à DAC unilateral da CI esquerda e único AVC na região parieto-occipital ${ }^{8}$. Aghaebrahim et al. (2013) também relatam que a paciente do seu estudo apresentou comprometimento sensório-motor no MS por DAC bilateral e único AVC na região parietal ${ }^{10}$. Ambas as pacientes dos estudos supracitados tinham idades semelhantes a paciente deste estudo, assim como com sintomas sensório-motor similares. Diferentemente dos estudos anteriores, a paciente deste estudo apresentou AVC nos dois hemisférios resultantes da DAC bilateral, fato que comprometeu fortemente seu estado clínico e prognóstico. Tendo isso, estes casos destacam a importância da aplicação de um protocolo para investigação da ocorrência de DAC por pequenos eventos traumáticos, como após procedimento odontológico, possibilitando o diagnóstico precoce.

Quanto ao tratamento medicamentoso, é recomendada para a suspeita de DAC a prescrição de anticoagulantes e antiplaquetários, sem evidência de superioridade de uma terapia sobre a outra ${ }^{22}$. Contudo, Santamarta-Fariña et al. (2004) relatam que apesar da utilização de anticoagulante, o paciente sofreu Acidente Isquêmico Transitório no hemisférico do mesmo lado da lesão anterior ${ }^{23}$. O mesmo aconteceu no estudo de Aghaebrahim et al. (2004), em que foram identificadas novas áreas de infarto nos exames de imagem mesmo após o manejo com terapia de anticoagulação na DAC bilateral após procedimento odontológico ${ }^{10}$. Neste mesmo estudo, os autores questionaram a eficácia dessa terapia dentro do quadro clínico da paciente. Ainda nesse contexto, Daou et al. (2017) mostraram que, dos casos analisados, há uma porcentagem com novos ou recorrentes eventos, apesar da administração de terapia antiplaquetária e anticoagulação no tratamento da $\mathrm{DAC}^{24}$. No entanto, investigação sobre a causa do AVCi na DAC através de mapas de territórios vasculares e etiologias obtidos por exames de imagem (angiografia, TC ou ressonância magnética) sugerem que a maioria dos infartos são provavelmente embólicos e não de origem hemodinâmica ${ }^{25}$. De acordo com esse mecanismo, as terapias anticoagulantes e antiplaquetárias parecem ser tratamento lógico e adequado no estágio inicial da DAC. Entretanto, sabe-se que em alguns casos com sintomas isquêmicos persistentes, as terapias citadas podem realmente não evitar novos AVC's, e a abordagem cirúrgica seria o mais recomendado ${ }^{3}$.

A literatura cita uma taxa de mortalidade de $5 \%$ associada às dissecções arteriais cervicais, sendo o prognóstico diretamente dependente da gravidade do evento isquêmico ${ }^{3}$. Essa porcentagem pode aumentar quando consideradas dissecções carotídeas, e ainda mais quando bilaterais. Campos et al. (2004), em estudo retrospectivo de 48 pacientes com dissecção arterial carotídea e vertebral, verificaram a ocorrência de DAC bilateral em três pacientes $(10,3 \%)$, sendo que dois deles vieram a óbito ${ }^{15}$. Desta forma, considerando o caso apresentado neste estudo com evolução da DAC em dois AVCs e incapacidade severa na alta, foi possível supor que a paciente poderia permanecer com algum nível de incapacidade funcional. No seguimento ambulatorial, após cinco meses da alta, a paciente encontrava-se com incapacidade severa (ERm 5) e assistida de forma contínua por toda equipe de reabilitação.

\section{CONCLUSÃO}

Em geral, o prognóstico após DAC apresenta boa recuperação funcional e baixa taxa de dissecção recorrente, complicações isquêmicas e hemorrágicas. Entretanto, não foi o encontrado no caso relatado, possivelmente por um diagnóstico tardio devido ao baixo índice de suspeita, resultado da soma do fator causal atípico e dos poucos achados iniciais. A ocorrência de uma dissecção cervical pode passar despercebida se não vier associada a dor relevante ou déficits neurológicos que motivem a busca do serviço médico, o que dificulta a avaliação da exata incidência dessa doença na população. Assim, este estudo destaca que fatores como traumas menores podem passar despercebidos ou pouco valorizados. Portanto, na presença de eventuais sintomas de alerta, faz-se necessário um meticuloso questionamento sobre as últimas atividades do indivíduo, assim como o estabelecimento de critérios diagnósticos com a solicitação de exames que possam corroborar a suspeita clínica. Outrossim, profilaxias podem ser adotadas na ocorrência de fatores causais como 
hiperextensão cervical prolongada ou movimentos súbitos da cervical. Diante do exposto, o diagnóstico da DAC é um desafio, em especial por se tratar de uma lesão de etiologia multifatorial. No entanto, seu reconhecimento precoce afetará de maneira direta o desfecho do paciente.

\section{REFERÊNCIAS BIBLIOGRÁFICAS}

1. Yaghi S, Maalouf N, Keyrouz SG. Cervical Artery Dissection: Risk Factors, Treatment, and Outcome; A 5-Year Experience From a Tertiary Care Center. Int J Neurosci [Internet]. $1^{\circ}$ de janeiro de 2012 [citado 25 de junho de 2020];122(1):40-4. Disponível em: https://doi.org/10. $3109 / 00207454.2011 .622453$

2. Lee VH, Brown RD, Mandrekar JN, Mokri B. Incidence and outcome of cervical artery dissection: a population-based study. Neurology. 28 de novembro de 2006;67(10): 1809-12.

3. Schievink W. Spontaneous Dissection of the Carotid and Vertebral Arteries. N Engl J Med [Internet]. 9 de agosto de 2001 [citado 24 de junho de 2020];345(6):467467. Disponível em: https://doi.org/10.1056/ NEJM200108093450616

4. Debette S, Leys D. Cervical-artery dissections: predisposing factors, diagnosis, and outcome. Lancet Neurol. julho de $2009 ; 8(7): 668-78$.

5. Brandt T, Orberk E, Weber R, Werner I, Busse O, Müller BT, et al. Pathogenesis of cervical artery dissections: Association with connective tissue abnormalities. Neurology [Internet]. 10 de julho de 2001 [citado $1^{\circ}$ de julho de 2020];57(1):24-30. Disponível em: https://n.neurology.org/content/57/1/24

6. Edmundson SP, Hirpara KM, Ryan RS, O'Malley T, O'Grady P. Delayed presentation of carotid artery dissection following major orthopaedic trauma resulting in dense hemiparesis. J Bone Joint Surg $\mathrm{Br}$. abril de 2009;91(4):536-9.

7. Biller José, Sacco Ralph L., Albuquerque Felipe C., Demaerschalk Bart M., Fayad Pierre, Long Preston H., et al. Cervical Arterial Dissections and Association With Cervical Manipulative Therapy. Stroke [Internet]. $1^{\circ}$ de outubro de 2014 [citado $1^{\circ}$ de julho de 2020];45(10):315574. Disponível em: https://www.ahajournals.org/doi/ full/10.1161/str.0000000000000016

8. Cerrato P, Giraudo M, Bergui M, Baima C, Grasso M, Rizzuto $A$, et al. Internal carotid artery dissection after mandibular third molar extraction. J Neurol. março de 2004;251(3):348-9.

9. Guillon B, Bousser MG. [Epidemiology and pathophysiology of spontaneous cervical artery dissection]. J Neuroradiol J Neuroradiol. dezembro de 2002;29(4):241-9.

10. Aghaebrahim A, Jankowitz BT, Jovin TG, Jadhav AP. Bilateral carotid dissections after a dental procedure: The role of stenting in unstable lesions. J Clin Neurosci [Internet]. $1^{\circ}$ de dezembro de 2013 [citado $1^{\circ}$ de julho de 2020];20(12):1778-80. Disponível em: http://www.sciencedirect.com/science/article/pii/S0967586813000532

11. Siwiec RM, Solomon GD. Bilateral carotid artery dissection after dental work. Headache. dezembro de 2007;47(10):1449-50.

12. Delgado MG, Riesco N, Murias E, Calleja S. Acute cervical artery dissection after a dental procedure due to a second inferior molar infection. BMJ Case Rep [Internet]. 2 de junho de 2015 [citado 23 de junho de 2020];2015. Disponível em: https://www.ncbi.nlm.nih.gov/pmc/articles/PMC4460314/

13. Provenzale JM, Sarikaya B. Comparison of test performance characteristics of MRI, MR angiography, and CT angiography in the diagnosis of carotid and vertebral artery dissection: a review of the medical literature. AJR Am J Roentgenol. outubro de 2009;193(4):1167-74.

14. Campos-Herrera CR, Scaff M, Yamamoto FI, Conforto AB. Spontaneous cervical artery dissection: an update on clinical and diagnostic aspects. Arq Neuropsiquiatr [Internet]. dezembro de 2008 [citado 25 de junho de 2020];66(4):922-7. Disponível em: http://www.scielo. $\mathrm{br} / \mathrm{scielo}$.php?script $=$ sci_abstract\&pid $=$ S0004-282X200 $8000600036 \&$ Ing $=e n \& n r m=i s o \& t \mid n g=e n$

15. Campos CR, Evaristo EF, Yamamoto FI, Puglia Jr P, Lucato LT, Scaff M. Dissecção espontânea cervical carotídea e verbal: estudo de 48 pacientes. Arq Neuropsiquiatr [Internet]. junho de 2004 [citado 28 de julho de 2020];62(2B):492-8. Disponível em: http://www. scielo.br/scielo.php?script $=$ sci_abstract $\&$ pid $=$ S0004282X2004000300021\&lng=en\&nrm=iso\&tlng=pt

16. Botelho T de S, Neto CDM, Araujo FLC de, Assis SC de. Epidemiologia do acidente vascular cerebral no Brasil. Temas em Saúde. 2016;16.

17. Norris JW, Beletsky V, Nadareishvili ZG. Sudden neck movement and cervical artery dissection. CMAJ Can Med Assoc ] [Internet]. 11 de julho de 2000 [citado 21 de julho de 2020];163(1):38-40. Disponível em: https:// www.ncbi.nlm.nih.gov/pmc/articles/PMC1232549/

18. Soo OY, Chan YL, Wong KS. Carotid artery dissection after prolonged head tilting while holding a newborn baby to sleep. Neurology. 11 de maio de 2004;62(9):1647-8.

19. Maroon JC, Gardner P, Abla AA, El-Kadi H, Bost J. "Golfer's stroke": golf-induced stroke from vertebral artery dissection. Surg Neurol. fevereiro de 2007;67(2):1638; discussion 168.

20. Gould DB, Cunningham K. Internal carotid artery dissection after remote surgery. Iatrogenic complications of anesthesia. Stroke. junho de $1994 ; 25(6): 1276-8$.

21. Zetterling M, Carlström C, Konrad P. Internal carotid artery dissection. Acta Neurol Scand. janeiro de $2000 ; 101(1): 1-7$.

22. Markus HS, Levi C, King A, Madigan J, Norris J, Cervical Artery Dissection in Stroke Study (CADISS) Investigators. Antiplatelet Therapy vs Anticoagulation Therapy in 
Cervical Artery Dissection: The Cervical Artery Dissection in Stroke Study (CADISS) Randomized Clinical Trial Final Results. JAMA Neurol. 01 de 2019;76(6):657-64.

23. Santamarta-Fariña E, Vaquero-Lorenzo F, López-García D, Cubillas-Martín H, Alonso-Gómez N, Gutiérrez-Julián JM. Aneurisma de carótida interna y estenosis carotídea preoclusiva bilateral. Angiología [Internet]. 2004 [citado 29 de julho de 2020];513-9. Disponível em: https:// pesquisa.bvsalud.org/portal/resource/pt/ibc-36104
24. Daou B, Hammer C, Mouchtouris N, Starke RM, Koduri $S$, Yang $S$, et al. Anticoagulation vs Antiplatelet Treatment in Patients with Carotid and Vertebral Artery Dissection: A Study of 370 Patients and Literature Review. Neurosurgery. 01 de 2017;80(3):368-79.

25. Lucas C, Moulin T, Deplanque D, Tatu L, Chavot D. Stroke patterns of internal carotid artery dissection in 40 patients. Stroke. dezembro de 1998;29(12):2646-8.

Autor Correspondente:

Dra. Chelin Auswaldt Steclan

chelin@unc.br

Editor:

Prof. Dr. Marcelo Riberto

Recebido: $17 / 08 / 2020$

Aprovado: 09/11/2020 